\title{
La rapide création du Parc Naturel marin de Mayotte
}

\section{Nicolas Legoff}

\section{(2) OpenEdition}

Journals

Édition électronique

URL : http://journals.openedition.org/echogeo/11808

DOI : 10.4000/echogeo. 11808

ISSN : 1963-1197

\section{Éditeur}

Pôle de recherche pour l'organisation et la diffusion de l'information géographique (CNRS UMR 8586)

\section{Référence électronique}

Nicolas Legoff, «La rapide création du Parc Naturel marin de Mayotte », EchoGéo [En ligne], Sur le Vif, mis en ligne le 06 avril 2010, consulté le 01 mai 2019. URL : http://journals.openedition.org/ echogeo/11808; DOI : 10.4000/echogeo.11808

Ce document a été généré automatiquement le 1 mai 2019.

\section{(c) (i) (9)}

EchoGéo est mis à disposition selon les termes de la licence Creative Commons Attribution - Pas d'Utilisation Commerciale - Pas de Modification 4.0 International 


\title{
La rapide création du Parc Naturel marin de Mayotte
}

\author{
Nicolas Legoff
}

\section{Introduction}

1 La création d'un parc marin est un fait remarquable pour le territoire français qui n'en compte qu'un seul : celui de la mer d'Iroise institué en 2007. Avec l'officialisation du Parc Naturel marin de Mayotte (PNMM) en janvier 2010, c'est donc un nouveau pas qui a été franchi. L'innovation va même largement plus loin puisque l'outre-mer français n'avait encore jamais été concerné par une telle démarche. Il peut paraître pour le moins surprenant de lancer cette procédure sur un territoire qui n'a même pas achevé sa départementalisation et dans lequel les problèmes de sous-développement sembleraient être la priorité.

2 Afin de comprendre les fondements de ce projet, il est utile de revenir sur le contexte et le processus politique qui motivent la naissance du PNMM. Etant donné les fortes singularités culturelles mahoraises, les usages dévolus à la zone protégée ne peuvent être simples et demandent aussi une analyse. A la lumière de ces éléments, la pertinence de la création d'un parc marin à Mayotte ne sera que plus claire, notamment en terme d'aide au développement de l'île.

\section{L'aboutissement rapide d'un processus}

3 Même si les comparaisons sont délicates en raison de l'unicité du Parc Naturel marin d'Iroise (3550 km²), c'est tout de même la rapidité qui prévaut dans le cas de Mayotte. Pour le parc breton, la démarche avait été laborieuse et l'aboutissement compliqué. Après bien des hésitations, des oppositions et des ajustements, il a fallu plus d'une décennie pour que la structure devienne opérationnelle. D'autres Parcs Naturels marins ${ }^{1}$ sont à l'étude mais risquent de se heurter à des difficultés de taille. On peut douter que tous dépassent un jour le stade de projet. Le jeu d'acteurs est en effet d'une telle complexité et 
les intérêts d'une telle valeur marchande qu'il y a fort à parier que les zonages proposés sur le littoral français seront systématiquement synonymes de blocages.

C'est peut-être la chance de Mayotte que d'être encore un territoire en devenir et d'accumuler des lacunes aussi bien administratives que sociales. En l'absence d'un tissu associatif véritablement organisé et d'une dynamique identitaire clairement identifiée, il est aisé pour Paris de légiférer sans soulever des oppositions trop vives. Ainsi, la signature du décret de création du Parc Naturel marin de Mayotte par le président Sarkozy lors de sa visite de janvier 2010 n'est que l'aboutissement logique d'une facile mise en place administrative. Si l'on tient compte de la date de constitution de la mission d'étude pour la création du PNMM en décembre 2007, seulement deux années de pilotage par la Direction de l'agriculture et de la forêt (D.A.F.) ont suffi pour boucler le dossier.

5 Cependant, à y regarder de plus près, cette rapidité pose question. Non pas sur la forme qui est juridiquement conforme, mais sur le fond et plus particulièrement sur la maturité des esprits quant à ce projet paradoxalement abstrait pour les acteurs locaux. Le code de l'environnement étant applicable à Mayotte, c'est lui qui a servi de cadre aux démarches successives depuis $2007^{2}$. Sous l'autorité directe du Préfet, la D.A.F. a d'abord mis en place une mission d'étude associant des personnels de l'Agence des aires marines protégées ainsi que des agents rattachés au Conseil général. Le premier objectif de la mission étant de connaître précisément la richesse naturelle (Gigou, 2009) et culturelle (Guezel, 2009) des eaux de Mayotte. C'est un préalable logique à la délimitation de l'aire à protéger et aux grands traits de la gestion attendue. Etablir l'architecture du conseil de gestion responsable du fonctionnement du parc a été l'ultime objectif à remplir par cette mission.

6 La Préfecture ${ }^{3}$ a bien entendu gardé la main sur les travaux préparatoires en présidant le comité de pilotage qui est entré en activité à partir de décembre 2008. Un panel représentatif des Collectivités publiques et des professionnels du lagon a eu pour rôle de donner son avis et son accord sur les diverses options suivies par la Mission d'étude. Symboliquement, lors de sa dernière assemblée en septembre 2009, le comité de pilotage a validé globalement l'ensemble des travaux réalisés et notamment les orientations de gestion qui seront déclinées en actions concrètes. Auparavant, et pour donner une tournure participative, des groupes de travail issus du comité de pilotage ont oeuvré sur trois axes forts et complémentaires: pêche et aquaculture, activités nautiques et touristiques, environnement et biodiversité. Toujours dans une optique d'ouverture, les travaux préparatoires se sont nourris de contacts par le truchement de réunions et de discussions décentralisées dans les villages.

$7 \quad$ Il est à craindre que l'échantillon touché par les rencontres et les réunions ne soit pas des plus représentatifs et la gestion proposée pour le parc en inadéquation avec les pratiques. Autrement dit, le risque est donc que les Mahorais contestent dans quelques années la présence d'un parc considéré comme mal adapté à la culture locale. En effet, s'il y a eu un consensus sur la philosophie du parc, il n'en demeure pas moins précaire ou du moins limité dans le temps. La contradiction relevée par Isabelle Autissier ${ }^{4}$ d'un désintérêt de la chose océanique par les Mahorais qui « ont encore à être sensibilisés à la mer » doit être gardée à l'esprit (Autissier, 2009). Avec la modernisation socioéconomique viendra le temps des rivalités territoriales sur le lagon et ses extensions hauturières nécessitant des réajustements... 


\section{Du bon usage de la zone protégée}

8 Pour l'heure, ce sont sept orientations de gestion qui sont au cœur du parc naissant (figure 1). Sept orientations qui ont été présentées lors de l'enquête publique, dernière obligation avant la signature du décret d'application. Là encore, ce n'est pas réellement la ferveur populaire qui caractérise cette étape. Le bilan n'est certes pas honteux avec environ 300 observations consignées sur les registres présents en mairies, mais la disparité entre les communes interroge. D'autant qu'un lourd dispositif de communication avait été mis en place et des permanents assignés à l'accueil des citoyens pendant plusieurs semaines.

Figure 1 - Les orientations proposées par le Comité de pilotage

\begin{tabular}{|c|c|}
\hline Les sept orientations de gestion & Exemples d'actions envisagées \\
\hline $\begin{array}{l}\text { a Faire de Mayatte un póle d'excellence en } \\
\text { matière de connaissance et de suivi des } \\
\text { ecosystemes marins tropicaux et de la } \\
\text { mangrove }\end{array}$ & $\begin{array}{l}\text { - Faite participer les professionnels de la póche, du tourisme } \\
\text { au suivi de fétat du milieu. } \\
\text { - Centraliser les études, étudier l'impact des pratiques. } \\
\text { - Suivre les espèces invasives, mener des études sur la } \\
\text { ressource, la péche récréative... }\end{array}$ \\
\hline $\begin{array}{l}\text { \% Obtenir une bonne qualté de f'eau dans le } \\
\text { lagon notamment par une gestion appropriée } \\
\text { des mangroves * }\end{array}$ & $\begin{array}{l}\text { - Etudier et suivre les impacts des apports telluriques. } \\
\text { - Organiser des actions de ramassage, airder a modifier les } \\
\text { pratiques responsables de l'érosion. } \\
\text { - Equiper des sites pour a der les praticues respectueuses. } \\
\text { - Expenimenter le rôle épurateur de la mangrove. }\end{array}$ \\
\hline $\begin{array}{l}\text { Développer une activité de péche } \\
\text { professionnelie hors du lagon, écolcgicuement } \\
\text { exemplaire et pourvoyeuse d'emplois et de } \\
\text { produits de la mer pour Mayotte s }\end{array}$ & $\begin{array}{l}\text { S et des ressources. } \\
\text { Concentration de Poissons. } \\
\text { rofessionnelle respectueux }\end{array}$ \\
\hline $\begin{array}{l}\text { Développer les filieres aquacoles } \\
\text { respectueuses de l'environnement en particulier } \\
\text { celes qui bénéticient directement aux } \\
\text { populations locales. }\end{array}$ & $\begin{array}{l}\text { quaculture (lfremer) } \\
\text { ns autres que le poisson en } \\
\text { rcialisation locale. } \\
\text { re blologicue } x\end{array}$ \\
\hline $\begin{array}{l}\text { Faire découvrir le milieu marin et sa } \\
\text { biodiversité grace à l'organisation des activités } \\
\text { de loisirs et a la professionnalisation des } \\
\text { acteurs cu tourisme * }\end{array}$ & $\begin{array}{l}\text { marin. } \\
\text { ue des activités } \\
\text { 15. }\end{array}$ \\
\hline $\begin{array}{l}\text { *. Pérenniser et valcriser les pratiques vivrières } \\
\text { et les savoirs traditionnels dans le cadre d'une } \\
\text { gestion precautionneuse du lagon } \%\end{array}$ & $\begin{array}{l}\text { s la gestion. } \\
\text { r-faire traditionnels }\end{array}$ \\
\hline $\begin{array}{l}\text { Protèger et meltre en valeur le patrimoine } \\
\text { naturel, de la mangrove aux espèces } \\
\text { océaniques, notamment par la formation et la } \\
\text { sensibilisation du plus grand nombre. }\end{array}$ & $\begin{array}{l}\text { - Réféchir à des moyens de protections ponctuelles. } \\
\text { - Renforcer l'éducation à l'environnement. } \\
\text { - Oévelopper des outils pédagogiques spécifiques } \\
\text { - Former les pêcheurs pour mieux gérer la ressource. }\end{array}$ \\
\hline
\end{tabular}

Source : Arnaud, 2009

9 Comme indiqué dans le document final de présentation (Arnaud, 2009), il n'y a pas de hiérarchie entre ces propositions. Du reste, il est délicat d'avancer des priorités et tous ces axes semblent à la fois nécessaires et urgents. C'est probablement là que se situera le premier défi de l'administration du Parc marin, à savoir sérier les engagements et ne pas se perdre dans les luttes d'influence.

10 L'éventail des actions est pratiquement exhaustif du fait que tous les acteurs socioéconomiques sont de près ou de loin concernés. La force des idées avancées pour le futur conseil de gestion est de ne pas être véritablement révolutionnaire mais plutôt de composer, ou pour mieux dire de recomposer, avec les pratiques coutumières comme l'est la pêche piroguière. Concernant cette dernière, la professionnalisation de la filière reste un chantier complexe à mener (Wickel, 2008) puisqu'elle repose encore grandement sur de l'informel: une flottille composée à $80 \%$ de pirogues, l'absence de structures portuaires, une coopérative qui ne draine qu'une infime partie des mises à terre... 
Tout se focalise donc vers la durabilité et des usages raisonnés sur une aire qui a eu des limites incertaines jusqu'au dernier moment. Ce n'est pas le moindre des paradoxes que d'avoir émis des orientations et tenté d'affiner des implications pratiques sans avoir la moindre idée de l'espace sur lequel les règles devront s'appliquer. Les hypothèses allaient de la limite stricte des eaux lagonaires à l'intégralité de la Zone économique exclusive (figure 2). Soit de $1100 \mathrm{~km}^{2}$ à plus de $69000 \mathrm{~km}^{2}$, ce qui n'est théoriquement pas sans modifier les tenants et aboutissants puisque le projet passe alors du local au régional.

Figure 2 - Les différentes limites possibles du Parc Naturel marin de Mayotte

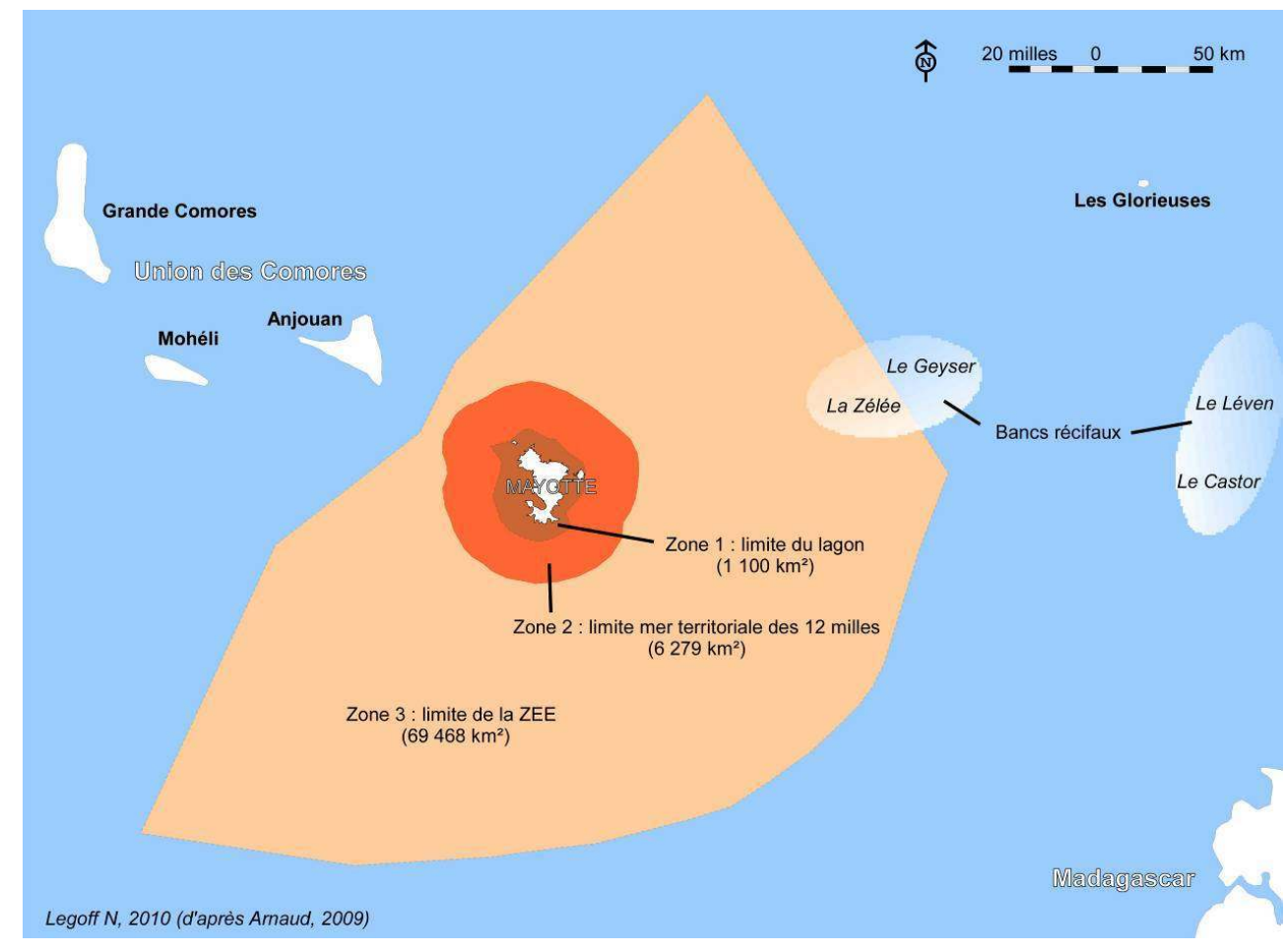

Source : Arnaud, 2009 ; réalisation : N. Legoff

Finalement, ce sont les limites de la ZEE qui ont été retenues dans le décret de création du PNMM (publié au J.O. le 21 janvier 2010). Pour remettre les choses en perspective, un tel zonage revient pratiquement à introduire un rapport de 1 à 200 entre la surface des îles mahoraises et le périmètre du parc (et un rapport de 1 à 20 avec le parc d'Iroise). C'est une situation parfaitement justifiable dès lors qu'il est souhaité que les activités halieutiques, y compris hauturières, soient intégrées dans les actions du parc ${ }^{5}$. Sans même cela, la présence de nombreux mammifères marins (baleines, dauphins, dugongs...) requiert de pouvoir raisonner sur des surfaces correspondant à leurs déplacements saisonniers.

Restent les interrogations sur les moyens matériels et humains qui seront alloués et qui feront du jeune parc un organisme efficace ou une pure vue de l'esprit.

\section{Une structure utile au développement de l'île?}

14 Il ne convient pas de développer les éléments qui font du futur $101^{\text {ème }}$ département français un territoire de pauvreté où les poches de sous-développement restent criantes 
notamment sous la forme de bidonvilles alimentés par les migrations clandestines (Taglioni, 2009). L'idée est plutôt de voir si l'instauration du PNMM peut être un moyen, parmi d'autres, pour catalyser le développement socioéconomique de l'île et ainsi combler le retard accumulé par rapport au département « voisin » de la Réunion qui sert souvent de référence.

Il est avant tout à espérer que les moyens déployés seront à la hauteur des ambitions avancées par le comité de gestion qui commencera par cibler les actions prioritaires dans un Plan d'action qui reste à officialiser. Il est d'ores est déjà question de la création d'une quarantaine d'emplois toutes fonctions confondues. Porter un jugement sur cette perspective est bien hasardeux sachant qu'il faudrait connaître les objectifs à moyen terme. Ceci étant, la comparaison est tentante avec ce qui existe déjà à Mayotte en aires protégées et en moyens déployés. Le lagon porte en effet deux réserves ${ }^{6}$ dont la gestion est confiée à la Brigade Nature créée en 2006. Cette dernière, placée sous la tutelle de l'office national de la chasse et de la faune sauvage compte cinq agents équipés d'une vedette rapide. Les conditions sont donc théoriquement réunies pour mener à bien les opérations de surveillance et d'interpellation des contrevenants.

Dans les faits, le bilan est largement plus mitigé car la réactivité des braconniers ${ }^{7}$ dépasse de loin celle de la brigade qui doit le plus souvent se contenter de constater les dégâts. Il s'agit pourtant de professionnels parfaitement formés et rompus à leurs prérogatives. En considérant les $69458 \mathrm{~km}^{2}$ du PNMM, la surveillance des eaux risque de rester symbolique si de lourds moyens matériels ne sont pas mis en jeu (flotte de patrouilleurs, recours à des survols...). Faire coïncider ZEE et limites du parc peut être considéré comme un signe fort de reprise en mains de ce territoire océanique par la France. Une sorte de rappel aux voisins qui n'ont jamais eu de cesse d'en revendiquer une partie (Taglioni, 2007)

Sur ces aspects matériels, le parc joue sa crédibilité et son degré d'efficacité. Parmi les effets induits sont d'abord attendues les retombées d'une pêche raisonnée qui s'apparente pour l'heure plutôt à un pillage en règle au cœur des réserves. Qu'il s'agisse des prises démersales à proximité des bancs récifaux voire de la barrière ou bien des captures pélagiques axées sur les thonidés, rien n'est véritablement contrôlé. Il serait inutile de structurer une véritable pêche artisanale dépassant le stade de la pirogue ou de la barque polyester si les stocks ne peuvent permettre un minimum de durabilité. Sans compter que la demande en produits de la mer ne peut qu'être exponentielle et que l'aquaculture naissante ${ }^{8}$ ne saurait répondre à la demande locale.

Indéniablement, il y a urgence à synchroniser les efforts de pêche au risque de tout perdre. Les thoniers-senneurs n'apporteront certainement pas de réel dynamisme à l'île car c'est tout juste s'ils font des escales techniques au port de Longoni et les droits des licences de pêche ne sont pas encore totalement acquis pour Mayotte ${ }^{9}$. Seule la pêche artisanale pourrait impulser des changements en apportant de la matière première à des industries agro-alimentaires qui font toujours défaut.

L'autre domaine pour lequel le PNMM pourrait être décisif est le tourisme. C'est tout bonnement une activité à inventer car elle est pour le moins confidentielle (BernardieTahir \& El-Mahaboubi, 2001). La destination de Mayotte reste en effet négligeable (moins de 40000 arrivées en 2009) et tournée vers un tourisme affinitaire lié à la présence de fonctionnaires métropolitains recevant leurs proches. Le manque d'équipements aéroportuaires est souvent mis en avant tout comme le manque de réceptifs hôteliers. 
Force est cependant de constater que rien n'a été véritablement tenté à Mayotte pour drainer des flux touristiques plus rémunérateurs et que l'amateurisme prévaut.

Le problème étant qu'il n'est aujourd'hui pas aisé de se faire une place dans l'océan Indien face à la Réunion, l'île Maurice, les Seychelles ou encore la prometteuse Madagascar. Mayotte a alors tout intérêt à cultiver sa différence et à proposer un produit singulier. En ce sens, l'étiquette de «Parc » est certainement une des meilleures, à la fois porteuse de qualité et d'authenticité. Soit de quoi construire une belle image de marque pour qui sait se servir de ce label. Aller en ce sens ne peut se faire sans une gestion participative et intégrée des ressources et des espaces (Breton, 2009) garante d'un équilibre durable.

Enfin, et ce n'est pas le moindre des enjeux à long terme, le parc a son rôle à jouer dans l'éducation à l'environnement de la jeune génération. Cette dernière étant celle qui subit et constate directement la dégradation de son environnement marin immédiat notamment à cause de la croissance urbaine (Ninon, 2007). Une sensibilisation à la fragilité du lagon ne permettra peut-être pas de restaurer les écosystèmes tant les processus polluants ont déjà fait des dégâts, mais une stabilisation serait déjà un objectif louable. Ce peut donc être un solide outil de communication visant à faire prendre conscience de la diversité et de la rareté des eaux mahoraises. Assigner une valeur au lagon est probablement le meilleur moyen pour qu'une surveillance environnementale efficace se mette en place, seule garante d'une pérennité et donc d'un développement local réel. Certains pensent même qu'il est possible d'aller encore plus loin et d'inscrire Mayotte et son lagon au Patrimoine mondial de l'UNESCO (Ministère de l'Ecologie, 2009).

\section{Conclusion}

Le Parc naturel marin de Mayotte a désormais une réalité juridique. De sa création restera l'idée d'un parfait déroulement de la procédure. Le défi qui s'annonce est conséquent car gérer une zone océanique de $69000 \mathrm{~km}^{2}$ est inédit, surtout s'il est tenu compte du gabarit modeste de l'île. Il ne faut pas attendre que le PNMM apporte à court terme de véritables changements ou impulse de nouvelles dynamiques socioéconomiques. Le comité de gestion aura certainement fort à faire pour fédérer les énergies et trouver les meilleurs leviers. Conclure, au titre de sa probable lente structuration, que le parc est un outil peu pertinent pour le développement de Mayotte serait aller un peu vite en besogne. De même, douter de l'adhésion sur le long terme des Mahorais à ce projet en raison de leur faible investissement actuel n'est pas fondé. Il se peut même que la période soit la plus propice pour faire passer un tel projet. Les responsables du Parc devront simplement se souvenir des circonstances de sa genèse et faire preuve de pragmatisme face à une société forcément évolutive. Mettre en place un parc marin à Mayotte dans une décennie ne serait certainement pas plus facile. Gageons que le PNMM et sa surface remarquable ne soit pas qu'un commode artifice pour permettre à la France d'arriver à ses objectifs quantitatifs avancés lors du Grenelle de l'environnement... 


\section{BIBLIOGRAPHIE}

Autissier I., 2009. Les Mahorais, étrangement, ont encore à être sensibilisés à la mer. Le Monde , 20 juin 2009.

Arnaud J-P. \& Al., 2009. Les propositions - pour un parc marin - Dossier de consultation du Parc naturel marin de Mayotte. Mission d'étude pour la création d'un parc naturel marin à Mayotte, Agence des aires marines protégées, $28 \mathrm{p}$.

Bernardie-Tahir N. \& El-Mahaboubi O., 2001. Mayotte : des parfums au tourisme. Les nouveaux enjeux du littoral. Les Cahiers d'Outre-Mer, Vol 216[En ligne] mis en ligne le 13 février 2008. URL : http://com.revues.org/index1137.html

Breton J-M, 2009. Aires protégées, gestion participative des ressources environnementales et développement touristique durable et viable dans les régions ultra-périphériques. Études caribéennes, 12/2009, Espaces et aires protégés. Gestion intégrée et gouvernance participative, [En ligne] mis en ligne le 4 septembre 2009.

Gigou A. \& Al., 2009. Un patrimoine naturel d'exception - Pour un parc naturel marin à Mayotte Dossier de consultation du Parc naturel marin de Mayotte. Agence des aires marines protégées, $60 \mathrm{p}$.

Guezel R. \& Al., 2009. Les hommes et l'océan - Pour un parc naturel marin à Mayotte - Dossier de consultation du Parc naturel marin de Mayotte. Agence des aires marines protégées, $72 \mathrm{p}$.

Ministère de l'Ecologie, 2009. Le Livre bleu des engagements au Grenelle de la mer : 138 engagements en faveur de la mer et du littoral. $71 \mathrm{p}$.

Ninon J., 2007. La dynamique urbaine à Mayotte : l'étalement de Mamoudzou et la « périphérisation » des centres petits-terriens. Les Cahiers d'Outre-Mer, n²40, p. 305-318.

Taglioni F, 2009. Mayotte s'ancre dans la république française. EchoGéo, Sur le vif, [En ligne] Mis en ligne le 01 juillet 2009. URL : http://echogeo.revues.org/index11277.html

Taglioni F., 2007. Les petits espaces insulaires au coeur des revendications frontalières maritimes dans le monde. L'Espace Politique, Vol.1, pp. 61-75, [En ligne] Mis en ligne en mars 2010. URL : http://espacepolitique.revues.org/index732.html

Wickel A., 2008. La crise du territoire à Mayotte : l'exemple de la pêche artisanale. L'espace politique, Vol. 6, p. 2-14.

\section{NOTES}

1. Lors du Grenelle de l'environnement, il a été question de 10 Parcs Naturels marins avant 2012 afin d'aboutir à une protection de $10 \%$ des surfaces océaniques françaises (20\% en 2020). Ainsi, en outre-mer, sont aussi pressentis la Martinique dans la zone Antilles, la Guyane, la Réunion et Saint-Pierre et Miquelon.

2. Loi du 14 avril 2006 relative aux parcs nationaux, aux parcs naturels marins, aux parcs naturels régionaux (loi n²006-436). 
3. La tutelle préfectorale est en fait double avec celle de Mayotte et celle de la Réunion au-delà de 6 milles nautiques.

4. Isabelle Autissier a fait partie des personnalités consultées lors du Grenelle de la mer et s'est plus particulièrement concentrée sur le groupe de «La délicate rencontre entre la terre et la mer » qu'elle a co-présidé. C'est dans ce cadre et celui d'une tournée en outre-mer qu'elle s'est rendue à Mayotte en juin 2009.

5. La ZEE ne permet cependant pas d'englober la totalité des bancs récifaux à commencer par le plus proche dont seule la partie Ouest entre dans les limites du parc. Le banc contigu du geyser s'en trouve ainsi exclu.

6. Réserve dite de la «Passe en $S$ » (1 380 hectares) et la réserve de la pointe de Saziley (800 hectares).

7. Les estimations de la Brigade nature avancent que 1000 tortues seraient capturées par an. L'infraction la plus courante étant le recours à des filets qui sont strictement interdits.

8. Environ 100 tonnes / an constituées d'espèces nobles facilement exportables telle l'ombrine.

9. Prochainement, l'administration des T.A.A.F. ne devrait plus être la seule bénéficiaire. Charge à la Commission régionale des pêches nautiques et aquacultures marines (COREPAM) de redistribuer des subventions aux pêcheurs.

\section{RÉSUMÉS}

Le second Parc Naturel marin français a été officialisé à Mayotte en janvier 2010. C'est à la fois le résultat d'une procédure rapide et d'une forte détermination politique. Il marque clairement la volonté de la France de gérer un large espace dans le sud-ouest de l'océan Indien même si les moyens d'action restent à déterminer. Au-delà de la protection environnementale, le Parc est perçu comme un outil utile à cette île encore synonyme de sous-développement. De la pêche au tourisme, bien des activités espèrent tirer profit de la dynamique enclenchée.

The 2nd french Marine Protected Area was officially created in Mayotte in January 2010, thanks both to a swift procedure and to a strong determined policy. It clearly shows the will of France to manage a broad area in south-west of the Indian Ocean even if its means of action are still to settle. Beyond environemental protection, the park is seen as a useful tool for an island still considered as emergent. From fishing to tourism, many activities hope to take advantage from the dynamic set in motion.

\section{INDEX}

Keywords : fishing, marine protected area, Mayotte, political process, sustainable development, tourism

Mots-clés : aire marine protégée, développement durable, Mayotte, pêche, processus politique, tourisme 


\section{AUTEUR}

\section{NICOLAS LEGOFF}

Nicolas Legoff est docteur \& chercheur associé au Laboratoire Géolittomer - UMR LETG 6554

(CNRS) / Université de Nantes. nicolas.legoff@univ-nantes.fr 